\title{
Impact of Doctoral Student Training Process Fit on Doctoral Students' Mental Health
}

\author{
Fulin $\mathrm{Li}^{1}$, Chuanyi Wang ${ }^{1, *}$ and Xiaoguang $\mathrm{Yue}^{2}$ \\ ${ }^{1}$ Institute of Education, Tsinghua University, Beijing, 100084, China \\ ${ }^{2}$ School of Sciences, European University Cyprus, Nicosia, 2404, Cyprus \\ *Corresponding Author: Chuanyi Wang. Email: wcy1985@tsinghua.edu.cn \\ Received: 30 November 2021 Accepted: 20 December 2021
}

\begin{abstract}
Background: Doctoral students have much higher risk of anxiety or depression than general population. Doctoral students worldwide are facing varying degrees of mental health risks. Method: Based on the survey data of 6,812 doctoral students worldwide in Nature in 2019, Probit and Logit models are used to explore the correlation between the fit of doctoral education and training process and the mental health of doctoral students. Results: (1) The training environment fit of doctoral students has a significant positive impact on their mental health. (2) The academic profession fit of doctoral students has a significant positive impact on their mental health. (3) The organizational culture fit of doctoral students has a significant positive impact on their mental health. (4) The financial support fit of doctoral students has a significant positive impact on their mental health. Conclusion: The higher the degree of doctoral students' training environment fit, academic profession fit, organizational culture fit, and financial support fit, the lower the possibility of anxiety or depression among doctoral students. The current research results can help reveal extensive factors that affect the mental health of doctoral students, facilitate the planning and development of effective intervention measures by universities, improve the fit of the doctoral education and training process, improve the mental health of doctoral students, and boost academic excellence.
\end{abstract}

\section{KEYWORDS}

Doctoral student training environment fit; doctoral student academic profession fit; doctoral student organizational culture fit; doctoral student financial support fit; mental health

\section{Introduction}

In the era of knowledge economy, those with a doctoral degree are vital to the social development, and there is a strong demand for researchers in the productive service industry and high-tech industries. At the same time, in most countries around the world, doctoral degree is a prerequisite for knowledge production and transformation in higher education institutions. However, doctoral students face much higher risk of anxiety or depression than the general population, and doctoral students worldwide are experiencing varying degrees of mental health risks. The University of California, Berkeley conducted a survey among 790 postgraduate students, and nearly half of the doctoral students in the survey met the criteria for depression [1]. The study by Levecque et al. [2] also found that $32 \%$ of doctoral students are at risk of developing a common mental illness, especially depression. This has affected the turnover rate of doctoral 
education to a certain extent. Several studies on doctoral students reveal that, depending on different disciplines and countries, the dropout rate for doctoral students is between $30 \%$ and $50 \%$ [3]. The COVID-19 epidemic has exacerbated the mental health challenges of doctoral students. A survey pointed out that the prevalence of major depressive disorder is as high as $36 \%$, and the prevalence of general anxiety disorder is as high as $43 \%$ [4]. The mental health of doctoral students has become one key issue to be urgently solved in the academic circle.

In the long doctoral career, the high research risk and daily hardship may breed feelings of loneliness and personal failure. Complicated experiments make working hours unpredictable, which will distort the balance between work and life and produce a sense of loss of control [5]. Academic completion brews under the factors of isolation, high expectations, and insomnia, which may contribute to debilitating depression, painful anxiety attacks, and even suicide attempts [6]. Fear of failure, low expectations for long-term career development and job security tend to make doctoral students anxious, and implementing multiple research projects at the same time is more likely to overload doctoral students lacking organizational skills, leading to burnout. Compared with other groups, the academic community faces greater risks than many people. Pressure from publishing, creating impact, winning funds, striving for tenure, attracting the public, showing up on social media, influencing policy, plus experiment failure, lonely jobs, and rigid hierarchies are all threats to the well-being of researchers including doctoral students [7].

In the traditional view, universities are a relatively low-stress field in society, and doctoral students are highly selected high-level talents, but psychological distress such as depression and anxiety is widespread in the process of doctoral training and has become a key factor influencing the completion time of doctoral study [8]. When doctoral students experience constant and frequent stress or depression in academic and daily life, it may make doctoral students abandon their studies. Such a high rate of personnel turnover will make it difficult for doctoral education to attract extraordinary talents, which will endanger the rate and quality of academic knowledge production. In addition, due to the original contribution to the basic scientific knowledge in the doctoral dissertation, the work of doctoral students themselves constitutes the main source of scientific progress. If there are a considerable number of doctoral students with mental health problems, it may affect the overall quality and quantity of personal research results, and bring greater costs to research institutions or research teams [9]. Therefore, it is imperative to pay attention to the mental health of doctoral students.

\section{Literature Review}

The World Health Organization's statistical report shows that globally, it is estimated that more than 300 million people suffer from depression, which is equivalent to $4.4 \%$ of the world's population. The number of people with common mental disorders is on the rise globally, especially in low-income countries. Depression affects people of all age groups in all walks of life. Life events such as poverty, unemployment, death of relatives or relationship breakdown, physical illness, and problems caused by alcohol and drug use all increase the risk of depression [10]. Depressive disorder has become the leading cause of non-fatal health loss in countries around the world. By comparison, doctoral students face higher risk of depression or anxiety, and the training process is full of factors that induce mental health among the group. In the group of doctoral students, depression, anxiety, and isolation are so widespread that some people refer to this experience as "doctoral depression" [11]. For doctoral students, the training process is often relatively closed and the communication groups are limited. In the limited development space, potential conflicts between doctoral students and their supervisors, research uncertainty, lack of financial support, difficulty in working-life balance, poor physical health, and individual personality characteristics may all trigger anxiety or depression among doctoral students to a certain extent. In this regard, in 2019, a survey article published in the journal Nature also showed that doctoral students have higher levels of depression, anxiety and stress than the general population [12]. Since better well-being of doctoral students contributes to the long-term 
benefits of academia and higher education [13], mental health of doctoral students urgently demands in-depth research in academia to provide evidence for effective interventions.

Then, what factors can induce mental health problems among doctoral students? Specifically, the factors leading to mental health among doctoral students can be divided into internal factors and external factors. In terms of internal factors, the mental health of doctoral students is mainly affected by the individual psychological characteristics of doctoral students. If doctoral students tend to compare with others and have perfectionist tendencies, they will be prone to "impostor syndrome." The academic circle refers to this phenomenon as "imposter phenomenon", which is used to refer to a false inner experience of intelligence [14]. This is a recognized disease, and many researchers face the disease risk at almost every stage of their careers. When doctoral students struggle with the feeling that they do not deserve to be a junior researcher at all, it may make the journey of doctoral study extremely difficult [15]. They fall into a panic state after comparing themselves with others unfavorably. In fact, in some fields, it may take years to achieve a breakthrough in publication, while in other cases, frequent publication is much easier [16]. However, it is very difficult to maintain personal identity in a highly turbulent and stressful career, and individuals will easily lose self-awareness [17]. For a researcher in training, maintaining mental health may be contradictory in terms of terminology. Many doctoral students are free to engage in the scientific field of their choice, at least in theory, have the opportunity to become a leading researcher in this field. However, frequent publication of papers, independent research, continuous application for funds, and meeting the needs of supervisors can cause a lot of emotional and mental tension, anxiety and pressure. These barriers will create a negative impact on the emotional health of doctoral students, especially when they do not anticipate such barriers or do not know how to overcome them [18].

At the same time, the deterioration of external environmental factors during the training of doctoral students often induces mental health problems among doctoral students. Researchers such as Pyhältö et al. [19] have identified four problem domains experienced by doctoral students: supervisors and academic communities, resources, domain-specific expertise, and general workflow. The frequency of meeting with supervisors, the difficulty of publishing doctoral dissertations, and the difficulty in balancing work-familydoctoral programs are correlated with depression and anxiety levels [20]. In the early stages of doctoral research, the importance of academic support for the mental health and well-being of doctoral students is obvious. Among them, the most important support provider is the supervisor, and positive supervisor support is crucial to responding to early challenges [21]. A supervisor is a guide for students to adapt to the academic community. An inharmonious relationship with the supervisor can also cause anxiety and stress [22]. For doctoral students, academic identity is the professional identity established by doctoral students in their cultivation, which is the core to the cultivation of doctoral students as young researchers [23]. When doctoral supervisors lack sufficient patience, experience, and research ability, they may not communicate well with doctoral students, which may lead to misunderstandings or false expectations. Although doctoral students may have made a deliberate choice before choosing to pursue a doctoral degree, if they cannot receive effective professional guidance, sufficient financial support, and develop ability to adapt to academic career, while their own autonomy cannot help them fully integrate into the academic community, the negative emotions of anxiety and depression may further affect the scientific research performance of doctoral students, and then trigger deeper psychological problems. In other words, the degree of fit between doctoral students and elements in the training process such as supervisors, academic communities, resource elements, academic careers, etc., will affect the mental health of doctoral students. When doctoral students can be reasonably engaged in the training process through a normal path, individual's mental health will be better; conversely, when doctoral students cannot be engaged in the doctoral training process in a timely and effective manner due to absence of many elements, the individual's well-being will decrease, inducing individual-level anxiety or depression. 
However, the existing research still has three deficiencies. First, data is incomplete, and the research sample is usually only applicable to a single university or region. International data is scarce and nonstandardized, and a larger scale of work is needed to understand the extent of mental health problems, and how specific aspects of doctoral students and the general academic environment contribute to these problems [24]. Second, there is no effective theoretical analysis framework that systematically sorts out and compares the factors affecting the mental health of doctoral students. If institutions and academic stakeholders ignore the structural driving factors of mental health problems among early career researchers, they will not be able to retain them [25]. Third, there may be an interaction between related variables that affect the mental health of doctoral students. The information contained in the variables is inevitably overlapping with each other, and it is impossible to simply correlate the training process variables of doctoral students with their mental health. Considering the above three aspects, in view of the central role of doctoral students in national technological competitiveness, to secure the quality of doctoral education and scientific research results of doctoral students, it is necessary to pay more attention to the environmental factors that induce doctoral students' anxiety or depression, and enhance the fit of doctoral education and training process. At present, there is no research that comprehensively discusses the entire range of stressors that induce doctoral students' bad emotions. It is still necessary to think about how to conduct research in a way that takes into account the entire doctoral education environment, and perform more in-depth and consistent research on the impact of doctoral education and training on the mental health of doctoral students [26]. Therefore, we should thoroughly conduct more comprehensive and systematic research on the factors that affect the mental health of doctoral students. This research is based on the survey data of 6,812 doctoral students in Nature in 2019, and introduces the fit theory in organization theory, with a view to revealing widespread factors that affect the mental health of doctoral students, which will help plan and develop effective intervention measures for universities, improve the fit of the doctoral education and training process, enhance the mental health of doctoral students, and boost academic excellence.

\section{Theoretical Analysis and Research Hypotheses}

Fit theory originates from organization theory, which believes that when there is consistency between personal values and their organization, tasks or social structure, then personal performance, satisfaction or happiness will grow [27]. The fit theory is a complex, multi-dimensional and dynamic concept, and the ever-changing educational situation requires the introduction of new theoretical perspectives and methods to study doctoral education and student experience. For this reason, Baker et al. [28] have demonstrated from the theoretical level the applicability of the popular theories of person-environment fit, personculture fit and person-vocation fit in organizational literature to the research of doctoral education, providing a new perspective for understanding the factors that cause the psychological dilemma of students in the process of doctoral education. Theoretically, by applying the fit theory to research, it is possible to examine the relationship between the mental health of doctoral students and the environment of their training process, reveal the multiple key factors that form a fit perception among doctoral students, as well as the antecedents and corresponding consequence of these processes. Accordingly, based on Ward et al. [29] theoretical analysis framework of doctoral education fit, this research focuses on investigating the impact of training environmental fit, academic profession fit, organizational culture fit, and financial support fit on the mental health of doctoral students.

\section{(1) The impact of cultivation environment fit on the mental health of doctoral students}

Training environment fit refers to the compatibility between individual doctoral students and the training environment. For doctoral students, they may be exposed to multiple environment aspects at the same time, mainly including the relationship between doctoral students and their supervisors, the fit of supervisor guidance and other faculty and staff's guidance, the fit with cooperative groups, and the fit with 
individual autonomy. Under normal circumstances, the relationship with the supervisor is an important factor that triggers the problem of doctoral education [30]. Brockman et al. [31] believe that the relationship between doctoral students and faculty members is a crucial determinant in the quality of postgraduate experience. The common understanding between supervisors and students is important for the well-being of doctoral students, and the expectation conflictions between the students and supervisors are regarded as one obstacle to the effective completion of the doctoral research [32]. The continuous destructive friction between students and the learning environment can lead to learning problems, and mental pain during education can have a negative impact and lead to withdrawal from learning [33]. In the training process of doctoral students, the relationship between doctoral students and their learning environment is mediated by experience with personal attributes, such as the students and their supervisors' previous learning experience, goals and strategies, and contextual factors [34], while supervisor guidance, supervisor experience and the frequency of meetings can effectively reduce students' emotional exhaustion [35]. Goplerud [36] studied the perception of social support and stress levels among psychology postgraduate students and found evidence that increased teacher support reduces stress perception. At the same time, as the highest level of education in all countries, doctoral education has a considerable level of openness, which is to develop the autonomy of doctoral students in an open learning environment to promote meaningful learning [37]. As doctoral students fit more into the training environment, the individual's happiness index will also continually increase, and the degree of mental health will be higher. In view of this, this research proposes hypothesis H1.

H1: Cultivation environmental fit has a significant positive impact on the mental health of doctoral students.

\section{(2) The impact of academic profession fit on the mental health of doctoral students}

The degree of academic professional fit mainly refers to the degree of suitability between doctoral students and the main activities of the academic community. For doctoral students, identity is the core of their doctoral study. They are working hard to develop another scholar's identity within the selected academic community because they need to adapt to, learn and use the specific discourse and thinking habits of the academic community [38]. According to previous research, the journey of a doctoral student can be seen as a continuous and meaningful process. In this process, the student needs to grow into a capable researcher and become an indispensable and valuable member of the academic community [39]. However, many doctoral students may feel isolated from the academic world or feel that they are not well fitted with the academic community to which they belong. The stress and fatigue experienced by doctoral students, at least to a certain extent, may stem from the mismatch between individual doctoral students and the academic community. At this time, by participating in the collective interaction of the academic community and by sharing the research of individual doctoral students, it will help them affirm that they are contributors to the academic community. Although individual doctoral students may have different motivations for participation, they are attracted to a professional academic community and begin to share their own research. In this way, they will perceive the common goals that arise within the academic community and improve the degree of fit between doctoral student individuals and the academic profession, thereby enhancing individual mental health. In view of this, this research proposes hypothesis H2.

$\mathrm{H} 2$ : The degree of academic profession fit has a significant positive impact on the mental health of doctoral students.

\section{(3) The impact of organizational culture fit on the mental health of doctoral students}

Organizational culture fit refers to the coordination between the study and life of doctoral students during their doctoral studies. It pays attention to the pace of learning and life of doctoral students in academic organizations, and emphasizes the degree to which doctoral students adapt to the work and life culture of academic organization in the school. Although the culture in academic organizations advocates freedom, this freedom is usually a kind of Janus-style freedom of thinking. It emphasizes the benefits of research freedom, but at the same time, this freedom also means that there is no clear boundary between 
work and leisure time. In particular, women face great pressure and find it difficult to achieve a balance between work and life [40]. In reality, academia often praises and rewards overwork, thus shaping the long-time work culture in the academic field. When students are unable to effectively adapt to the work culture during the doctoral study period, and have difficulty in achieving a balance between work and life, they will feel uneasy and lose control of the doctoral study life. In other words, doctoral students need to develop the ability to control academic research work, effectively grasp the pace of work and rest time to adapt to the learning and work culture in academic organizations. Occupational health research focusing on work control and mental health found that work control has a positive effect on mental health [41]. The higher the degree of work control, the lower the emotional cost of people and the higher the degree of mental health [42]. In view of this, this research proposes research hypothesis H3.

H3: Organizational culture fit has a significant positive impact on the mental health of doctoral students.

\section{(4) The impact of financial support fit on the mental health of doctoral students}

The degree of economic support fit mainly refers to the degree to which the economic and financial resources provided inside and outside the school meet the development needs of doctoral students. Excessive studies have proved that financial stress is generally related to depression and anxiety [43,44]. The same is true for doctoral students. Financial status may affect the mental health of doctoral researchers. Lack of financial resources is usually a major challenge faced by doctoral researchers $[45,46]$. Research based on the financial support of doctoral students found that researchers who received funding during the doctorate period perform better than self-funded researchers. Compared with funding for research projects, doctoral funding during their studies and their entire career can exert a positive impact on research performance [47]. Seen from this perspective, giving doctoral students sufficient financial support will not only create a positive impact on the mental health of doctoral students during their degree studies, but will also extend the welfare effects brought about by a healthy mentality to career of doctoral students. In view of this, this research proposes research hypothesis H4.

H4: Financial support fit has a significant positive impact on the mental health of doctoral students.

\section{Data, Variables and Analysis Methods}

\section{(1) Data source}

The data used in this research comes from the 2019 Nature Global Doctoral Survey, which is provided in English, Spanish, Chinese, French, and Portuguese, and has a high degree of international participation. The annual questionnaire covers topics such as doctoral students' study experience, career support, and maintenance responsibility, and asks questions about mental health among all interviewees for the first time. A total of 6812 valid questionnaires were collected. Where, European effective samples account for $36 \%$, Asian effective samples account for $28 \%$, North America and Central America effective samples account for 27\%, Africa, South America and Australasia effective samples account for $9 \%$. On the whole, the survey data covers the major doctoral training countries in the world, the data survey process conforms to academic norms, and the samples are highly representative.

\section{(2) Descriptive statistics of fit measurement items and variables}

In accordance with the index connotation of the doctoral training process, this research further divides the question items to measure the doctoral training environment fit, the academic profession fit, the organizational culture fit, and the financial support fit (see Table 1 for details). First of all, 7 items including supervisor recognition, supervisor-student relationship, supervisor guidance, guidance from others, career guidance and advice, cooperation opportunity, research autonomy, etc., indicate the training environment fit of doctoral students. Therefore, the above 7 items are used to measure the training environment fit of doctoral students. Second, the 3 items including the ability to present research results at conferences, academic conference participation, and academic publication are close to doctoral students' academic profession fit in 
connotation, which can reflect academic profession fit of doctoral students. Third, the 5 items including worklife balance, vacation time, working hour, health benefit, and social environment essentially reflect the adaptability of doctoral students in the school's organizational work culture. Therefore, the above 5 items are used to measure the organizational culture fit of doctoral students. Finally, fund sufficiency, subsidy, and teaching task can reflect the ability of doctoral students to seek financial support. Therefore, the above 3 items are used to measure the financial support fit of doctoral students.

Table 1: The dimensions of doctoral student training process fit and their measurement

\begin{tabular}{ll}
\hline Dimensions of doctoral student training process fit & Measurement item \\
\hline & Supervisor recognition \\
& Supervisor-student relationship \\
Training environmental fit & Supervisor guidance \\
& Guidance from others \\
& Career guidance and advice \\
& Cooperation opportunity \\
& Autonomy \\
\hline \multirow{2}{*}{ Academic profession fit } & Ability to present research results at conferences \\
& Academic conference participation \\
\hline & Academic publication \\
\hline Organizational culture fit & Work-life balance \\
& Vacation time \\
& Working hour \\
& Health benefit \\
& Social environment \\
\hline & Fund sufficiency \\
\hline
\end{tabular}

The dependent variable is the mental health of doctoral students. This study uses the questionnaire to characterize the respondent's response to "Have you ever sought help for anxiety or depression caused by $\mathrm{PhD}$ study?" Select "yes" and a value of 0 is assigned, indicating the presence of mental health problems. Select "No" and a value of 1 is assigned, which means that doctoral students have better mental health. The individual characteristic variables of doctoral students include (1) gender, male is assigned a value of 1 , female is assigned a value of 0 ; (2) age, 18-24 is assigned a value of 1, 25-34 is assigned a value of $2,35-44$ is assigned a value of 3,45-54 is assigned a value of 4, 55-64 is assigned a value of 5 , and 65 years old and above is assigned a value of 6 . A value of $1-6$ is assigned to indicate increasing age; (3) doctoral study motivation, academic motivation is assigned a value of 1 , and non-academic motivation is assigned a value 0; (4) maintenance responsibility. If there are children or elderly people in the family who need to take care of, the value is 1 . Otherwise, the value is 0 . Among the variables of the doctoral training process, doctoral students' training environment fit, academic profession fit, organizational culture fit, and financial support fit involve a total of 18 items, which measure the satisfaction perception towards supervisor-student relationship, supervisor guidance, supervisor recognition, guidance from others, fund sufficiency, working hour, social environment, scientific research autonomy, number of 
publications, cooperation opportunity, subsidy, vacation time, health benefit, teaching task, academic conference participation, ability to present research results at conferences, work-life balance, career guidance and advice, etc., with a value of 1-7 to characterize increasing fit between doctoral student individuals and the training process. In addition, the study will also generate country-specific dummy variables to control the influence of country factors on the mental health of doctoral students.

Specifically, in the group without mental health problems, men account for $54.7 \%$, in the group with mental health problems, men account for $38.9 \%$, and there is a higher proportion of male doctors with mental health than female doctors. In terms of age, academic motivation and maintenance responsibility, doctoral students without mental health have higher average number than those with mental health problems. Regarding the 18 explanatory variables involved in the training process of doctoral students, doctoral students without mental health problems have higher self-assessment scores than those with mental health problems. Take the supervisor-student relationship as an example. The average self-evaluation score of doctoral students without mental health problems is 5.347, while that of doctoral students with mental health problems is only 4.590. Variable names and descriptive statistics are shown in Table 2.

Table 2: Descriptive statistics of main variables

\begin{tabular}{|c|c|c|c|c|c|c|}
\hline \multirow[t]{2}{*}{ Variable name } & \multicolumn{3}{|c|}{$\begin{array}{l}\text { Without mental health } \\
\text { problem }\end{array}$} & \multicolumn{3}{|c|}{$\begin{array}{l}\text { With mental health } \\
\text { problem }\end{array}$} \\
\hline & $\mathrm{N}$ & Mean & $\begin{array}{l}\text { Standard } \\
\text { deviation }\end{array}$ & $\mathrm{N}$ & Mean & $\begin{array}{l}\text { Standard } \\
\text { deviation }\end{array}$ \\
\hline Gender & 4045 & 0.547 & 0.498 & 2402 & 0.389 & 0.488 \\
\hline Age & 4067 & 2.032 & 0.625 & 2422 & 1.989 & 0.523 \\
\hline Academic motivation & 4000 & 0.835 & 0.371 & 2357 & 0.783 & 0.412 \\
\hline Maintenance responsibility & 4031 & 0.218 & 0.413 & 2392 & 0.181 & 0.385 \\
\hline Fund sufficiency & 3968 & 4.644 & 1.930 & 2383 & 4.442 & 1.975 \\
\hline Working hour & 4053 & 4.650 & 1.663 & 2416 & 4.120 & 1.740 \\
\hline Social environment & 4064 & 4.810 & 1.682 & 2421 & 4.322 & 1.794 \\
\hline Research autonomy & 4060 & 5.556 & 1.440 & 2420 & 5.139 & 1.632 \\
\hline Supervisor recognition & 4044 & 5.113 & 1.736 & 2423 & 4.379 & 1.976 \\
\hline Supervisor-student relationship & 4041 & 5.347 & 1.693 & 2420 & 4.590 & 2.004 \\
\hline Supervisor guidance & 4014 & 4.743 & 1.810 & 2395 & 4.142 & 1.974 \\
\hline Guidance from others & 3924 & 4.815 & 1.702 & 2350 & 4.337 & 1.849 \\
\hline Cooperation opportunity & 4016 & 4.872 & 1.696 & 2408 & 4.356 & 1.854 \\
\hline Academic publication & 3857 & 3.893 & 1.821 & 2345 & 3.410 & 1.798 \\
\hline Subsidy & 4002 & 4.411 & 1.906 & 2410 & 4.158 & 1.950 \\
\hline Vacation time & 4008 & 4.610 & 1.870 & 2386 & 4.045 & 1.969 \\
\hline Health benefit & 3893 & 4.190 & 1.967 & 2350 & 3.893 & 2.009 \\
\hline Teaching task & 3349 & 4.552 & 1.630 & 2043 & 4.334 & 1.663 \\
\hline Academic conference participation & 4017 & 5.175 & 1.644 & 2399 & 4.772 & 1.817 \\
\hline Ability to present research results at conferences & 3938 & 5.123 & 1.657 & 2350 & 4.693 & 1.813 \\
\hline Work-life balance & 4055 & 4.221 & 1.646 & 2418 & 3.517 & 1.664 \\
\hline Career guidance and advice & 3981 & 3.861 & 1.731 & 2385 & 3.344 & 1.755 \\
\hline
\end{tabular}




\section{(3) Analysis method}

In the process of research, it was discovered that there are several variables involved in the cultivation of doctoral students, and the information contained in the variables inevitably overlaps with each other. There is a strong correlation between the variables, and the distribution of samples involves complicated laws. Therefore, this study adopts the principal factor method to reduce the dimension and simplify the structure of the doctoral students' training process variables, reduces multiple variables to a few relatively weakly correlated, brand-new comprehensive factors, integrates the initial variables as much as possible, and attempts to explain key information superimposed by variables. Since the dependent variable mental health is a dichotomous variable, the Probit model is used for regression analysis, and the possible heteroscedasticity problem of the model is analyzed through the likelihood ratio test. In order to test the robustness of the analysis results, this study also takes the Logit model and the OLS model for regression analysis.

\section{Empirical Analysis Results}

(1) Reliability and validity test of measurement items for doctoral students' training process fit

This study first uses the Cronbach reliability test to verify the internal consistency between the previous measurement items of doctoral students' training environment fit, academic profession fit, organizational culture fit, and financial support fit. Specifically, the Cronbach reliability test coefficient of 7 items including supervisor recognition, supervisor-student mentorship, supervisor guidance, guidance from others, career guidance and advice, cooperation opportunity, and research autonomy is 0.87 , which exceeds the acceptable threshold of 0.7. Afterwards, selection was made according to the principle that the Cronbach reliability test coefficient of the selected index should exceed 0.7 , academic publication was deleted, the two items of ability to present research results at conferences and academic conference participation have a Cronbach reliability test coefficient of 0.93. Similarly, after deleting the two items of health benefit and social environment, the Cronbach reliability test coefficient of the three items of worklife balance, vacation time, and working hour is 0.77 . Finally, after deleting the teaching task item, the Cronbach reliability test coefficient of the two items including fund sufficiency and subsidy is 0.73 . The above results show that the selected items have good internal consistency in measuring doctoral students' training environment fit, academic profession fit, organizational culture fit, and financial support fit.

Meanwhile, the KMO value in Table 3 is $0.845>0.7$, and the $P$ value of Bartlett's test is 0.000 , which significantly rejects the null hypothesis that the indicators are not correlated. This indicates that the factor analysis method is very suitable. Hence, this study uses the principal factor method to analyze the remaining 14 items, and a better distinguishable factor structure is obtained. According to the Kaiser criterion, 4 factors with characteristic roots greater than 1 were extracted. These 4 factors explain $68.75 \%$ of the total variance and result in the rotation factor loading (see Table 4).

Table 3: KMO test value, Bartlett sphere test

\begin{tabular}{lll}
\hline \multicolumn{2}{c}{ Kaiser-meyer-olkin measure of sampling adequacy (KMO) } & 0.845 \\
\hline \multirow{3}{*}{ Bartlett test of sphericity } & Chi-square & 41837.987 \\
& Degrees of freedom & 91 \\
& $p$-value & 0.000 \\
\hline
\end{tabular}


Table 4: Rotation factor loading

\begin{tabular}{|c|c|c|c|c|}
\hline Variable & Factor 1 & Factor 2 & Factor 3 & Factor 4 \\
\hline Supervisor recognition & 0.846 & & & \\
\hline Supervisor-student relationship & 0.842 & & & \\
\hline Supervisor guidance & 0.817 & & & \\
\hline Guidance from others & 0.653 & & & \\
\hline Career guidance and advice & 0.573 & & & \\
\hline Cooperation opportunity & 0.563 & & & \\
\hline Degree of autonomy & 0.525 & & & \\
\hline Ability to present research results at conferences & & 0.928 & & \\
\hline Academic conference participation & & 0.920 & & \\
\hline Work-life balance & & & 0.803 & \\
\hline Vacation time & & & 0.797 & \\
\hline Working hour & & & 0.731 & \\
\hline Fund sufficiency & & & & 0.865 \\
\hline Subsidy & & & & 0.842 \\
\hline
\end{tabular}

Specifically, the factor analysis results are consistent with the post-screening measurement items of doctoral students' training environment fit, academic profession fit, organizational culture fit, and financial support fit, indicating that the selected measurement items have good reliability and validity. Factor 1 contains 7 items, the most important of which is that doctoral students expect to gain recognition from their supervisors, develop a benign interactive relationship with supervisors, receive academic guidance from supervisors and others, receive career-related guidance and advice to gain broad cooperation opportunity. It shapes the autonomy of doctoral students as independent researchers. These contents fully express the degree of fit between doctoral students with the cultivation environment. Therefore, consistent with the previous content, we name it "doctoral students' training environment fit". Factor 2 contains two items, which mainly characterizes the ability of doctoral students to present research results in academic conferences, and emphasizes the academic participation behavior of doctoral students. These items are connotatively close to the degree to which doctoral students fit the academic profession. Therefore, consistent with the previous content, the factor is named "doctoral students' academic profession fit". Factor 3 contains 3 items, mainly expressing that the cultivation culture must support the physical and mental development of doctoral students, helping them to achieve a balance between academic and life, and get sufficient vacation time after work. These factors essentially reflect the adaptability of doctoral students to the school's organizational work culture. Therefore, consistent with the previous content, the factor is named "doctoral students' organizational culture fit". Factor 4 contains 2 items, mainly indicating the sufficiency of scientific research funds and subsidies in supporting the academic career and daily life of doctoral students, which are basic material guarantee for doctoral students to carry out scientific research. Therefore, consistent with the previous content, the factor is named "doctoral students' financial support fit" (see Table 3).

\section{(2) The impact of doctoral training process fit on the mental health of doctoral students}

Table 5 reports the benchmark regression results of the impact of doctoral students' training environment fit, academic profession fit, organizational culture fit and financial support fit on their mental health. The estimation results of the Probit model indicate that the impact of doctoral students' training environment 
fit, academic profession fit, organizational culture fit and financial support fit on the mental health is significantly positive at $1 \%$ level. It suggests that when doctoral students are more fitted to the training environment with higher adaptation to academic occupations and organizational culture and greater financial support, they have better mental health. Further measurement of the marginal effects of the four factors found that, given other variables, the marginal effects of doctoral students' training environment fit, academic profession fit, organizational culture fit, and financial support fit are respectively 0.078, $0.027,0.070$, and 0.018 , which are all significantly positive at the $1 \%$ level. This shows that for doctoral students, the training environment fit has the highest effect on doctoral students. This is consistent with the results of previous studies. The training environment involving supervisor relationship, supervisor guidance, autonomy, etc., has the crucial effect on doctoral students' mental health, and construction of a good relationship has a positive effect on the healthy development of doctoral students. Second, the organizational cultural fit of doctoral students also has a high degree of influence on the mental health of doctoral students. It can also be seen from Nature's survey that the doctoral student group is not satisfied with work-life balance, working hour and vacation time. This is also an important factor that causes emotional stress, anxiety or depression among doctoral students. Furthermore, the academic profession fit represented by doctoral students' academic conference participation and ability to present research results at conferences has a positive impact on the mental health of doctoral students. When they can adapt to the academic ecology and present their own research results, the individuals have better mental health. Finally, although financial support fit of doctoral students has a significant impact on their mental health, it has the lowest impact among the four factors. This shows that for doctoral students, although financial support is also very important, more important is the construction of the invisible supervisor-student relationship, the balance of life and research work, and the opportunity and ability to adapt to academic career development. Seen from the results of other explanatory variables, under the premise of controlling country factors, male doctors have better mental health. Research by Kurtz-Costes et al. [48] also found that female doctors suffer more pressure than male doctors.

Table 5: Regression results of the impact of training environment fit on the mental health of doctoral students

\begin{tabular}{llll}
\hline & $\begin{array}{l}\text { Probit } \\
\text { Mental health }\end{array}$ & $\begin{array}{l}\text { Logit } \\
\text { Mental health }\end{array}$ & $\begin{array}{l}\text { OLS } \\
\text { Mental health }\end{array}$ \\
\hline Cultivation environment fit & $0.216^{* * *}$ & $0.355^{* * *}$ & $0.078^{* * *}$ \\
& $(0.019)$ & $(0.031)$ & $(0.007)$ \\
Academic profession fit & $0.078^{* * *}$ & $0.128^{* * *}$ & $0.028^{* * *}$ \\
Organizational culture fit & $(0.018)$ & $(0.030)$ & $(0.007)$ \\
Economic support fit & $0.200^{* * *}$ & $0.328^{* * *}$ & $0.071^{* * *}$ \\
& $(0.018)$ & $(0.030)$ & $(0.006)$ \\
Motivation for doctoral study & $0.051^{* * *}$ & $0.086^{* * *}$ & $0.018^{* * *}$ \\
Maintenance responsibility & $(0.019)$ & $(0.031)$ & $(0.007)$ \\
Age & 0.043 & 0.066 & 0.016 \\
& $(0.047)$ & $(0.077)$ & $(0.017)$ \\
& 0.052 & 0.087 & 0.018 \\
& $(0.050)$ & $(0.082)$ & $(0.017)$ \\
& 0.004 & 0.008 & 0.001 \\
& $(0.036)$ & $(0.059)$ & $(0.012)$ \\
\hline
\end{tabular}




\begin{tabular}{llll}
\hline Table 5 (continued) & & & \\
\hline & Probit & Logit & OLS \\
& Mental health & Mental health & Mental health \\
\hline Gender & $0.381^{* * *}$ & $0.627^{* * *}$ & $0.134^{* * *}$ \\
& $(0.037)$ & $(0.061)$ & $(0.013)$ \\
Country dummy variable & control & control & control \\
-cons & $0.252^{* * *}$ & $0.410^{* * *}$ & $0.594 * * *$ \\
& $(0.091)$ & $(0.150)$ & $(0.032)$ \\
\hline $\mathrm{N}$ & 5341 & 5341 & 5341 \\
Pseudo $\mathrm{R}^{2} / \mathrm{R}^{2}$ & 0.074 & 0.074 & 0.094 \\
\hline
\end{tabular}

Note: $* * *$ indicate significance at the levels of $1 \%$, respectively, and the robust standard errors are in parentheses.

Table 6 reports the estimated results of the grouping regression of the main developed countries involved in the sample. The estimation results of the Probit model show that doctoral students' training environment fit has a significant positive impact on the mental health of doctoral students in the United States, the United Kingdom, Germany, France, Australia and South Korea; doctoral students' academic profession fit has a significant impact on the mental health of US doctoral students; doctoral students' organizational cultural fit has a significant positive impact on the mental health of doctoral students in the United States, the United Kingdom, Germany, France, Canada, Japan, etc.; doctoral students' financial support fit has a significant positive impact on mental health of British doctoral students.

Table 6: Grouping regression estimation results of developed countries (Probit)

\begin{tabular}{lllllllll}
\hline & $(1)$ & $(2)$ & $(3)$ & $(4)$ & $(5)$ & $(6)$ & $(7)$ & $(8)$ \\
& United States & United Kingdom & Germany & France & Australia & Canada & Japan & South Korea \\
\hline Cultivation environment fit & $0.210^{* * *}$ & $0.310^{* * *}$ & $0.247^{* * *}$ & $0.305^{* *}$ & $0.430^{* * *}$ & 0.067 & 0.171 & $0.556^{* *}$ \\
& $(0.038)$ & $(0.070)$ & $(0.068)$ & $(0.125)$ & $(0.118)$ & $(0.110)$ & $(0.145)$ & $(0.228)$ \\
Academic profession fit & $0.097^{* *}$ & 0.072 & -0.000 & -0.003 & -0.037 & -0.169 & 0.173 & 0.214 \\
& $(0.038)$ & $(0.072)$ & $(0.068)$ & $(0.153)$ & $(0.132)$ & $(0.125)$ & $(0.159)$ & $(0.158)$ \\
Organizational culture fit & $0.192^{* * *}$ & $0.335^{* * *}$ & $0.255^{* * *}$ & $0.346^{* *}$ & 0.104 & $0.260^{* *}$ & $0.297 *$ & 0.253 \\
& $(0.036)$ & $(0.069)$ & $(0.070)$ & $(0.153)$ & $(0.113)$ & $(0.122)$ & $(0.170)$ & $(0.202)$ \\
Economic support fit & 0.015 & $0.202^{* * *}$ & 0.110 & -0.120 & 0.080 & -0.184 & -0.096 & -0.344 \\
& $(0.040)$ & $(0.070)$ & $(0.077)$ & $(0.144)$ & $(0.122)$ & $(0.120)$ & $(0.166)$ & $(0.257)$ \\
Control variable & Control & Control & Control & Control & Control & Control & Control Control \\
cons & -0.219 & 0.379 & 0.395 & -0.332 & $1.445 * * *$ & $2.083 * * *$ & 0.131 & $-1.880^{*}$ \\
& $(0.146)$ & $(0.254)$ & $(0.485)$ & $(0.654)$ & $(0.500)$ & $(0.540)$ & $(0.723)$ & $(1.052)$ \\
\hline $\mathrm{N}$ & 1267 & 417 & 404 & 107 & 148 & 150 & 73 & 63
\end{tabular}

Note: ${ }^{* *}$, and $* * *$ indicate significance at the levels of $10 \%, 5 \%$, and $1 \%$, respectively, and the robust standard errors are in parentheses. 
Models (1)-(4) in Table 7 report the estimated results of grouping regression of the main developing countries involved in the sample, including four emerging market countries in the world: China, India, South Africa and Brazil. The estimation results of the Probit model show that doctoral students' training environment fit has a significant positive impact on the mental health of doctoral students in China, India and South Africa; the academic profession fit has a significant positive impact on the mental health of South African doctoral students; organizational culture fit has a significant positive impact on the mental health of doctoral students in the four countries; financial support fit has a significant positive impact on the mental health of doctoral students in India and South Africa.

Table 7: Regression estimation results of developing country grouping and cross-border flow grouping (Probit)

\begin{tabular}{lllllll}
\hline & $(1)$ & $(2)$ & $(3)$ & $(4)$ & $(5)$ & $(6)$ \\
& China & India & South Africa & Brazil & Study in the country & Cross-border flow \\
\hline Cultivation environment fit & $0.153^{* *}$ & $0.236^{* * *}$ & $0.487^{*}$ & -0.037 & $0.194^{* * *}$ & $0.245^{* * *}$ \\
& $(0.063)$ & $(0.072)$ & $(0.267)$ & $(0.137)$ & $(0.024)$ & $(0.030)$ \\
Academic profession fit & -0.017 & 0.069 & $0.823^{* * *}$ & 0.106 & $0.107^{* * *}$ & 0.025 \\
& $(0.054)$ & $(0.067)$ & $(0.302)$ & $(0.117)$ & $(0.023)$ & $(0.030)$ \\
Organizational culture fit & $0.126^{* *}$ & $0.224^{* * *}$ & $1.170^{* * *}$ & $0.471^{* * *}$ & $0.198^{* * *}$ & $0.207^{* * *}$ \\
& $(0.056)$ & $(0.070)$ & $(0.330)$ & $(0.130)$ & $(0.023)$ & $(0.030)$ \\
Economic support fit & 0.023 & $0.169^{* *}$ & $0.835^{* *}$ & -0.007 & $0.068^{* * *}$ & 0.034 \\
& $(0.059)$ & $(0.066)$ & $(0.329)$ & $(0.126)$ & $(0.024)$ & $(0.031)$ \\
Control variable & $c 0 n t r o l$ & control & control & control & control & control \\
cons & -0.021 & -0.361 & 1.281 & 0.143 & $0.283^{* *}$ & 0.221 \\
& $(0.254)$ & $(0.413)$ & $(1.203)$ & $(0.698)$ & $(0.112)$ & $(0.163)$ \\
\hline N & 610 & 384 & 45 & 107 & 3390 & 1951 \\
Pseudo $\mathrm{R}^{2}$ & 0.035 & 0.093 & 0.452 & 0.125 & 0.079 & 0.081 \\
\hline Note: $*{ }^{* *}$, and $* * *$ & indicate significance at the levels of $10 \%, 5 \%$, and $1 \%$, respectively, and the robust standard errors are in parentheses.
\end{tabular}

Models (5)-(6) in Table 7 report the heterogeneous impact of doctoral training process fit on doctoral students studying for doctoral degrees in their home countries and doctoral students studying for doctoral degrees in foreign countries based on whether doctoral students flow across borders. The estimation results show that for doctoral students studying in their home countries, doctoral students' training environment fit, academic profession fit, organizational culture fit, and economic support fit all have significant positive effects on their mental health. For doctoral students studying for a doctoral degree in foreign countries, doctoral students' training environment fit and organizational culture fit have a significant positive impact on their mental health.

\section{(3) Robustness test}

First, seen from the estimation results of the Probit and Logit models, the coefficients of the two models have been consistently estimated under the premise that the robust standard errors have been used. Moreover, the robust standard errors are very close to the ordinary standard errors, indicating that the model is correctly set. Second, the standard Probit and Logit models assume that the perturbation term is homoscedastic. As the doctoral students in the sample data come from different countries and regions, to better test the robustness of the estimation results and eliminate the interference of heteroscedasticity, it is possible to perform a likelihood-ratio test. If the null hypothesis is accepted, it is considered that the Probit model of 
homoscedasticity can be used. Otherwise, the Probit model of heteroscedasticity should be used. The estimation result suggests that the $P$ value of the likelihood ratio test is 0.661 , so the null hypothesis of homoscedasticity can be accepted, indicating that the estimation result of the benchmark Probit model is reliable and robust. Finally, the estimation results of Probit model, Logit model and OLS model are basically the same, which further demonstrates the robust analysis results.

\section{Conclusion and Discussion}

At the stage of doctoral education, the demand for mental health support keeps increasing. In this study, through theoretical analysis, the factors involved in the training of doctoral students are defined as doctoral students' training environment fit, academic professional fit, organizational culture fit, and financial support fit. The training process factors that affect the mental health of doctoral students are subject to dimensionality reduction and simplification, and it is found that the results are consistent with the theoretical division results. The doctoral student training process can be defined with four factors of training environment fit, academic profession fit, organizational culture fit and financial support fit. The estimation results of the Probit model, the Logit model and the OLS model reveal that under higher doctoral students' cultivation environment fit, academic profession fit, organizational culture fit, and economic support fit, doctoral students have better mental health. This also indicates that the use of fit theory can effectively predict multiple factors that affect the mental health of doctoral students. Further calculation of the marginal effects of the four factors found that according to the magnitude of the marginal effects on the mental health of doctoral students, the four factors are ranked as doctoral students' training environment fit, organizational culture fit, academic profession fit, financial support fit. There are also several issues for discussion in the analysis:

First, training process fit that affects the mental health of doctoral students is a multi-level fit, rather than a single-level fit. Intervention measures at the school management level should comprehensively consider feasibility, cost, and acceptability, and coordinate with multiple parties to promote the healthy development of doctoral students. In reality, there are complex interactions between multiple factors that affect doctoral students' mental health. It is precisely for this reason that this study uses factor analysis to reduce and simplify the factors involved in the training process of doctoral students. It integrates all the variable factors involved in the cultivation process of doctoral students as much as possible, and attempts to understand the key information co-existing in the doctoral cultivation process variables. Based on it, this study found that under higher fit between doctoral students and their training process, there is lower possibility that doctoral students will experience anxiety or depression. Put it bluntly, doctoral students' training environment fit, academic profession fit, organizational culture fit, and economic support fit are all embedded in the doctoral education ecology, which act together on the mental health of doctoral students. If the degree of fit is reduced in one aspect, it may affect the fit of other dimensions, which in turn affects the mental health of doctoral students. For example, when a doctoral student is facing financial pressure, he may seek more teaching assistant jobs, and the increase in teaching tasks will bring about conflicts in the roles of doctoral students, so it is difficult to balance life and work, with working hours prolonged and vacation time shortened. The superimposed influence of various factors will lead to disorderly study and research life of the doctoral students, and then affect their mental health. At present, few studies consider the work-life balance of doctoral students even though students at this level also encounter many of the same challenges as faculty and staff while pursuing different degree studies $[49,50]$. Such superimposed comprehensive impact means that it is difficult for university administrators to use linear thinking to solve the mental health dilemma of doctoral students in one time. It thus requires supervisors, families and individual doctoral students in doctoral education ecology to comprehensively understand the mental health problems of doctoral students, improve the fit between doctoral students and the training process in order to improve the mental health of doctoral students. In view of this, it is recommended that research policymakers learn from the concept of total quality management, shape the 
doctoral student support system through entire-process system construction, explore systematic system design from training links, career development, organizational culture, and economic support, strive to improve doctoral students' understanding towards school, department policies and degree standards, and improve doctoral students' fit with the training process.

Second, compared to the index fit at the explicit level, more attention should be paid to the construction of invisible relationships, the accumulation of tacit knowledge, and the improvement of general ability. According to the analysis on the marginal effects of the four factors in this study, doctoral students' training environment fit, organizational culture fit, academic profession fit have a greater impact on the mental health of doctoral students. Doctoral education is permeated with widespread culture of overtime work, and various financial aids tend to reward doctoral students who work overtime on a continuous basis. That is to say, although the financial support provided by schools and supervisors has a positive effect on the mental health of doctoral students, more attention should be paid to the influence of implicit factors on the mental health of doctoral students. Judging from the variables included in the first three factors, the improvement of doctoral students' training environment fit depends on the establishment of good interpersonal relationships between doctoral students, their supervisors and others, so that they receive sufficient academic guidance and cooperation opportunities, and develop individual autonomy in academic research life. However, both the construction of a benign relationship and the development of autonomy require more tacit knowledge and general ability support. Just as a Finnish doctoral student called "Help us make science more humane!" in this Nature's survey. This suggests that university administrators should design a personalized curriculum guidance system for supervisors and doctoral students by establishing joint supervisor, adding corresponding professional instructors in life and psychology, thus helping doctoral students adapt to the complicated path of study. At the same time, policymakers can encourage colleges and universities to set up effective early warning mechanisms. When students have a low degree of fit with their main supervisor, which may induce anxiety or depression among doctoral students, supervisor should be changed in time to avoid potential harm and loss. In addition, the construction of invisible relationships requires research policymakers to provide doctoral students with extensive opportunities for academic participation, support and encourage doctoral students to communicate with colleagues in the academic world, thus promoting the professional socialization of doctoral students. On this basis, doctoral students can better handle the work-life balance, adapt to the learning and work culture during the doctoral period, expand wiser academic participation, meet the needs for the environment, organizational culture, and academic profession development in doctoral training process, reduce psychological anxiety and improve mental health.

Third, by grouping regression of the main countries involved in the sample, it is found that doctoral students' training environment fit, academic profession fit, organizational culture fit, and economic support fit have varying effects on the mental health of doctoral students in various countries. Where, the two factors of doctoral students' training environment fit and organizational culture fit have a high predictive effect on the mental health of doctoral students in most countries. For doctoral students in various countries, the first problem that cannot be avoided during their doctoral study is the relationship with their supervisors and other instructors. Good supervisor-student cooperation has a positive promotion effect on the mental health of doctoral students, especially under the current background of big flow of doctoral students internationally. In Nature's survey sample this time, doctoral students who migrate across borders accounted for $36.10 \%$. For the cross-border doctoral students, under the dual pressure of cross-culture and highly challenging academic research life, it is even more necessary to build a good academic relationship to deal with potential anxiety or depression. In this process, another important factor is whether doctoral students can adapt to the organizational culture of the school to achieve a balance between work and life, which is also an important factor in the formation of a positive mental attitude. Schools should assist doctoral students in setting scientific working hours, guarantee their 
vacation time, help them form a stable and reasonable pace of life and work, and reduce the probability of anxiety or depression. In addition, due to differences in the economic, social and cultural aspects of various countries, the ability and willingness to provide doctoral students with academic career development opportunities, financial assistance, and mental health guidance may vary. Countries should pay attention to the universal fit factors. On the basis of this, it is necessary to focus on balancing the impact of specific factors on the mental health of doctoral students. For example, for doctoral students in developing countries, relevant countries and university organizations may be required to provide more sufficient funds and subsidies. At the same time, taking into account the potential differences due to cultural factors, it is necessary to pay attention to the particularity of the cultural attributes of doctoral students in various countries and take personalized intervention measures. All in all, taking into account the differences in countries. In particular, under the current background with continuous spread of COVID-19, the differences in epidemic prevention concepts, measures, results, and propaganda among various countries contribute to de-globalization and populism to a certain extent, which greatly hinders the flow and learning of doctoral students. It may easily cause disconnection between doctoral students and training process, which in turn induces mental health problems among doctoral students. This objectively requires policymakers to fully consider the economic and cultural development in the country of origin of doctoral students, combine the elements of the doctoral training process in respective country to create a comprehensive international doctoral student support system, scientifically implement policies, provide more effective academic and life guidance suggestions for international doctoral students, help them effectively adapt to and integrate into the social culture of the country of entry.

To sum up, in view of the outstanding contributions of the doctoral student group to academic research, mental health of doctoral students needs to be treated with caution. Existing research has also proved the importance of mental health of doctoral students. The contribution of this research is to enrich the existing theories explaining the mental problems of doctoral students, and make a more reliable and steady organization, comparison and generalization of the factors affecting the mental health training process of doctoral students. Specifically, this research uses the fit theoretical analysis framework to effectively distinguish the stressors that affect the mental health of doctoral students, conducts grouping analysis according to the country and mobility, etc., explores the possible heterogeneity effects, and verifies the appropriateness of using fit theory to analyze the mental health problems of doctoral students, which improves the academia and policymakers' awareness towards mental health problems of doctoral students, and provides an empirical basis for selecting priority options in policy intervention.

\section{Research Limitations}

This study has certain limitations. First, this study focuses on the impact of the overall fit between doctoral students and the training process on their mental health, which helps to reveal the factors that affect the mental health of doctoral students, enhance the university's awareness that the doctoral training process affects the mental health of doctoral students, and provide priority options for policy intervention. However, the study has not yet explored the mutual influence of the multiple factors involved in the doctoral student training process, such as supervisor-student relationship, supervisor guidance, career guidance and advice, financial support, work and life. The effects of various factors on the health and well-being of doctoral students are complicated. It is necessary to supplement effective interview data to effectively explain the impact of training process fit on the mental health of doctoral students. Second, anxiety or depression degree may be different among different groups, so the interpretation of binary variable estimation results must be treated with caution. In the future, it is necessary to study in more detail the relationship between the doctoral training process fit and the severity of doctoral students' anxiety or depression. Finally, the data used in this study is cross-sectional data, which cannot reveal the dynamic change process of doctoral students' mental health. In the future, we can continually track and 
collect survey data of relevant doctoral students to explore the varying impact of doctoral training process fit on their mental health, then achieve dynamic adjustment of the training process to improve the fit between doctoral students and their training process.

Availability of Data and Materials: https://figshare.com/s/74a5ea79d76ad66a8af8.

Funding Statement: The authors received no specific funding for this study.

Conflicts of Interest: The authors declare that they have no conflicts of interest to report regarding the present study.

\section{References}

1. Sohn, E. (2016). Mental health: Caught in a trap. Nature, 539(7628), 319-321. DOI 10.1038/nj7628-319a.

2. Levecque, K., Anseel, F., de Beuckelaer, A., van der Heyden, J., Gisle, L. (2017). Work organization and mental health problems in PhD students. Research Policy, 46(4), 868-879. DOI 10.1016/j.respol.2017.02.008.

3. Stubb, J., Pyhältö, K., Lonka, K. (2012). The experienced meaning of working with a $\mathrm{PhD}$ thesis. Scandinavian Journal of Educational Research, 56(4), 439-456. DOI 10.1080/00313831.2011.599422.

4. Chirikov, I., Soria, K. M., Horgos, B., Jones-White, D. (2020). Undergraduate and graduate students' mental health during the COVID-19 pandemic. UC berkeley: Center for studies in higher education. https://escholarship.org/uc/ item/80k5d5hw.

5. Editorial (2018). A PhD state of mind. Nature Cell Biology, 20(4), 363. DOI 10.1038/s41556-018-0085-4.

6. Gewin, V. (2012). Mental health: Under a cloud. Nature (London), 490(7419), 299-301. DOI 10.1038/nj7419-299a.

7. Reay, D. (2018). I'd whisper to my student self: You are not alone. Nature (London), 557(7704), 160-161. DOI 10.1038/d41586-018-05080-6.

8. Barry, K., Woods, M., Warnecke, E., Stirling, C., Martin, A. (2018). Psychological health of doctoral candidates, study-related challenges and perceived performance. Higher Education Research and Development, 37(3), 468483. DOI 10.1080/07294360.2018.1425979.

9. Marais, G. A. B., Shankland, R., Haag, P., Fiault, R., Juniper, B. (2018). A survey and a positive psychology intervention on French PhD student well-being. International Journal of Doctoral Studies, 13, 109-138. DOI $10.28945 / 3948$.

10. World Health Organization Depression and Other Common Mental Disorders: Global Health Estimates (WHO) (2017). http://go.nature.com/2ksfnhg.

11. Harrington, K. (2018). Harness the power of groups to beat the 'PhD blues'. Nature, 559(7712), 143-144. DOI $10.1038 / \mathrm{d} 41586-018-05589-\mathrm{w}$.

12. Nature (2019). The mental health of PhD researchers demands urgent attention. Nature, 575(7782), 257-258. DOI 10.1038/d41586-019-03489-1.

13. E Park, K., Sibalis, A., Jamieson, B. (2021). The mental health and well-being of master's and doctoral psychology students at an urban Canadian university. International Journal of Doctoral Studies, 16, 429-447. DOI 10.28945/4790.

14. Clance, P. R., Imes, S. A. (1978). The imposter phenomenon in high achieving women: Dynamics and therapeutic intervention. Psychotherapy: Theory, Research \& Practice, 15(3), 241-247. DOI 10.1037/h0086006.

15. Keogh, M. (2020). How to shake off the 'impostor' fears that plague your PhD studies. Nature. DOI 10.1038/ d41586-020-02401-6.

16. Gawusu, S. (2019). The PhD student's dilemma. Nature. DOI 10.1038/d41586-019-03881-x.

17. Seaborne, R. (2020). A person before a PhD: Understanding and combatting an academic identity crisis. Nature, 578(7794), 327-328. DOI 10.1038/d41586-020-00306-y.

18. Gould, J. (2014). Mental health: Stressed students reach out for help. Nature, 512(7513), 223-224. DOI 10.1038/ nj7513-223a.

19. Pyhältö, K., Toom, A., Stubb, J., Lonka, K. (2012). Challenges of becoming a scholar: A study of doctoral students' problems and wellbeing. ISRN Education, 7(2), 1-12. DOI 10.5402/2012/934941. 
20. Liu, C., Wang, L., Qi, R., Wang, W., Jia, S. et al. (2019). Prevalence and associated factors of depression and anxiety among doctoral students: The mediating effect of mentoring relationships on the association between research self-efficacy and depression/anxiety. Psychology Research and Behavior Management, 12, 195-208. DOI 10.2147/PRBM.

21. Bean, T. W., Readence, J. E., Barone, D. M., Sylvester, T. (2004). An Interpretive study of dctoral mentoring in literacy. Mentoring \& Tutoring: Partnership in Learning, 12(3), 371-381. DOI 10.1080/030910042000275963.

22. di Pierro, M. (2007). Excellence in doctoral education: Defining best practices. College Student Journal, 41(2), 368.

23. Cotterall, S. (2015). The rich get richer: International doctoral candidates and scholarly identity. Innovations in Education and Teaching International, 52(4), 360-370. DOI 10.1080/14703297.2013.839124.

24. Nature (2019). Being a PhD student shouldn't be bad for your health. Nature, 569(7756), 307. DOI 10.1038/ d41586-019-01492-0.

25. Forrester, N. (2021). Mental health of graduate students sorely overlooked. Nature, 595(7865), 135-137. DOI 10.1038/d41586-021-01751-z.

26. Mackie, S., Bates, G. (2019). Contribution of the doctoral education environment to PhD candidates' mental health problems: A scoping review. Higher Education Research and Development, 38(3), 565-578. DOI 10.1080/ 07294360.2018.1556620.

27. Edwards, J., Billsberry, J. (2010). Testing a multidimensional theory of person-environment fit. Journal of Managerial Issues, 22(4), 476-493.

28. Baker, V., Pifer, M. (2015). Antecedents and outcomes: Theories of fit and the study of doctoral education. Studies in Higher Education (Dorchester-on-Thames), 40(2), 296-310. DOI 10.1080/03075079.2013.823936.

29. Ward, A., Brennan, N. (2020). Developing a student-doctoral education fit analytical model to assess performance. Studies in Higher Education (Dorchester-on-Thames), 45(7), 1448-1460. DOI 10.1080/03075079.2018.1545758.

30. Bruce, C., Stoodley, I. (2013). Experiencing higher degree research supervision as teaching. Studies in Higher Education (Dorchester-on-Thames), 38(2), 226-241. DOI 10.1080/03075079.2011.576338.

31. Brockman, J., Nunez, A., Basu, A. (2010). Effectiveness of a conflict resolution training program in changing graduate students style of managing conflict with their faculty advisors. Innovative Higher Education, 35(4), 277-293. DOI 10.1007/s10755-010-9142-z.

32. Vähämäki, M., Saru, E., Palmunen, L. (2021). Doctoral supervision as an academic practice and leader-member relationship: A critical approach to relationship dynamics. The International Journal of Management Education, 19(3), 100510. DOI 10.1016/j.ijme.2021.100510.

33. Pyhältö, K., Stubb, J., Lonka, K. (2009). Developing scholarly communities as learning environments for doctoral students. The International Journal for Academic Development, 14(3), 221-232. DOI 10.1080/ 13601440903106551.

34. Murphy, N., Bain, J., Conrad, L. (2007). Orientations to research higher degree supervision. Higher Education, 53(2), 209-234. DOI 10.1007/s10734-005-5608-9.

35. Devine, K., Hunter, K. (2016). Doctoral students' emotional exhaustion and intentions to leave academia. International Journal of Doctoral Studies, 11, 35-61. DOI 10.28945/3396.

36. Goplerud, E. N. (1980). Social support and stress during the first year of graduate school. Professional Psychology, 11(2), 283-290. DOI 10.1037/0735-7028.11.2.283.

37. Moss, G., Kubacki, K. (2007). Researchers in higher education: A neglected focus of study? Journal of Further and Higher Education, 31(3), 297-310. DOI 10.1080/03098770701425006.

38. McAlpine, L., Amundsen, C. (2009). Identity and agency: Pleasures and collegiality among the challenges of the doctoral journey. Studies in Continuing Education, 31(2), 109-125. DOI 10.1080/01580370902927378.

39. Stubb, J., Pyhältö, K., Lonka, K. (2011). Balancing between inspiration and exhaustion: PhD students' experienced socio-psychological well-being. Studies in Continuing Education, 33(1), 33-50. DOI 10.1080/ $0158037 X .2010 .515572$. 
40. Appel, M., Dahlgren, L. (2003). Swedish doctoral students' experiences on their journey towards a PhD: Obstacles and opportunities inside and outside the academic building. Scandinavian Journal of Educational Research, 47(1), 89-110. DOI 10.1080/00313830308608.

41. de Lange, A., Taris, T., Kompier, M., Houtman, I., Bongers, P. (2004). The relationships between work characteristics and mental health: Examining normal, reversed and reciprocal relationships in a 4-wave study. Work and Stress, 18(2), 149-166. DOI 10.1080/02678370412331270860.

42. Vanroelen, C., Levecque, K., Louckx, F. (2009). Psychosocial working conditions and self-reported health in a representative sample of wage-earners: A test of the different hypotheses of the demand-control-support-model. International Archives of Occupational and Environmental Health, 82(3), 329-342. DOI 10.1007/s00420-008-0340-2.

43. Fitch, C., Hamilton, S., Bassett, P., Davey, R. (2011). The relationship between personal debt and mental health: A systematic review. Mental Health Review Journal, 16(4), 153-166. DOI 10.1108/13619321111202313.

44. Meltzer, H., Bebbington, P., Brugha, T., Farrell, M., Jenkins, R. (2013). The relationship between personal debt and specific common mental disorders. European Journal of Public Health, 23(1), 108-113. DOI 10.1093/eurpub/cks021.

45. Metcalfe, J., Levecque, K., Wilson, S. (2018). Exploring wellbeing and mental health and associated support services for postgraduate researchers. https://re.ukri.org/documents/2018/mental-health-report.

46. Van der Haert, M., Arias Ortiz, E., Emplit, P., Halloin, V., Dehon, C. (2014). Are dropout and degree completion in doctoral study significantly dependent on type of financial support and field of research? Studies in Higher Education (Dorchester-on-Thames), 39(10), 1885-1909. DOI 10.1080/03075079.2013.806458.

47. Horta, H., Cattaneo, M., Meoli, M. (2018). PhD funding as a determinant of $\mathrm{PhD}$ and career research performance. Studies in Higher Education (Dorchester-on-Thames), 43(3), 542-570. DOI 10.1080/03075079.2016.1185406.

48. Kurtz-Costes, B., Andrews Helmke, L., Ülkü-Steiner, B. (2006). Gender and doctoral studies: The perceptions of Ph. D. students in an American university. Gender and Education, 18(2), 137-155. DOI 10.1080/09540250500380513.

49. Zheng, S. Y., Jiang, S. P., Yue, X. G., Pu, R., Li, B. Q. (2019). Application research of an innovative online education model in big data environment. International Journal of Emerging Technologies in Learning, 14(8), 125-138. DOI 10.3991/ijet.v14i08.10404.

50. Schwoerer, K., Antony, M., Willis, K. (2021). PhDlife: The effect of stress and sources of support on perceptions of balance among public administration doctoral students. Journal of Public Affairs Education, 27(3), 326-347. DOI $10.1080 / 15236803.2021 .1876474$. 